\title{
O papel da escola na saúde bucal de crianças em idade escolar: Estudo Piloto
}

\author{
The role of the school environment in the oral health of schoolchildren: A pilot study \\ El papel del entorno escolar en la salud bucal de los niños en edad escolar: Un estudio piloto
}

Recebido: 29/04/2021 | Revisado: 01/06/2021 | Aceito: 04/06/2021 | Publicado: 18/06/2021

Thais Dias dos Santos

ORCID: https://orcid.org/0000-0002-7226-7519

Universidade do Grande Rio, Brasil

E-mail: diasthaissantos2@gmail.com

Luisa Catarina Porfírio de Sousa

ORCID: https://orcid.org/0000-0002-7160-259X

Universidade do Grande Rio, Brasil

E-mail: luisa.catarinaps95@gmail.com

Leila Chevitarese de Oliveira

ORCID: https://orcid.org/0000-0002-5037-8787

Universidade do Grande Rio, Brasil

E-mail: 1chevitarese@unigranrio.edu.br

Katiana Vidal

ORCID: https://orcid.org/0000-0001-8289-8966 Universidade do Grande Rio, Brasil

E-mail: katiana.vidal@unigranrio.edu.br

\begin{abstract}
Resumo
Objetivo: Avaliar o papel da escola na educação em saúde bucal de crianças em idade escolar, abrangendo toda a equipe pedagógica. Métodos: A pesquisa exploratória se deu através da aplicação de questionários direcionados para a equipe educacional da escola, a fim de obter os dados no formato de entrevista. Os dados foram transportados para o Google formulários, seguido da geração de uma planilha e gráficos fornecidos pelo próprio software. Resultados: Foi avaliado um total de 11 voluntários pertencentes a equipe escolar. As porcentagens encontradas foram altas no que diz respeito ao tema: atuação da escola como parceira dos pais na instrução de higiene bucal, somada aos resultados otimistas quanto ao conhecimento prévio e autocuidado dos entrevistados. Conclusão: Foi possível perceber que a escola desempenha um papel importante na educação em saúde bucal, e que atua como uma importante ferramenta de políticas públicas para o controle e combate às doenças cárie e periodontal, tornando-se indispensável, em especial no grupo de polarização.
\end{abstract}

Palavras-chave: Saúde bucal; Educação em saúde; Prevenção; Odontologia.

\begin{abstract}
Objective: To evaluate the role of the school in oral health education of schoolchildren, covering the entire pedagogical team. Methods: the exploratory research took place through the application of questionnaires directed to the school's educational team, in order to obtain the data in the interview format. The data was transported to Google Forms, followed by the creation of a spreadsheet and graphs provided by the software itself. Results: A total of 11 volunteers belonging to the school team were evaluated. The percentages found were high with regard to the theme: the school's role as a partner of parents in oral hygiene instruction added to the optimistic results regarding the previous knowledge and self-care of the respondents. Conclusion: it was possible to realize that the school plays an important role in oral health education and that it acts as an important public policy tool for the prevention and control of caries and periodontal diseases, becoming indispensable, especially in the polarization group.
\end{abstract}

Keywords: Oral health; Health education; Prevention; Dentistry.

\section{Resumen}

Objetivo: Evaluar el papel de la escuela en la educación en salud bucal de los niños en edad escolar, abarcando a todo el equipo pedagógico. Métodos: La investigación exploratoria se realizó mediante la aplicación de cuestionarios dirigidos al equipo educativo de la escuela, con el fin de obtener los datos en formato de entrevista. Los datos fueron transportados a formularios de Google, seguidos de la generación de una hoja de cálculo y gráficos proporcionados por el propio software. Resultados: Se evaluó a un total de 11 voluntarios pertenecientes al equipo escolar. Los porcentajes encontrados fueron altos con respecto al tema: el desempeño de la escuela como socio de los padres en la instrucción de la higiene bucal, sumado a los resultados optimistas en cuanto al conocimiento previo y el autocuidado de los entrevistados. Conclusión: Se pudo percibir que la escuela juega un papel importante en la educación en salud bucal, y que actúa como una importante herramienta de políticas públicas para el control y combate de caries y enfermedades periodontales, volviéndose indispensable, especialmente en el grupo de polarización.

Palabras clave: Salud bucal; Educación para la salud; Prevención; Odontología. 


\section{Introdução}

A prevenção e a manutenção da saúde são somente conseguidas por meio do processo educativo, estabelecendo mudanças de hábitos essenciais. Desta forma, para haja a transformação de hábitos deletérios em hábitos compatíveis com a saúde, é importante a utilização de mecanismos educativos que forneçam informações básicas em saúde de forma permanente e continuada, dando autonomia aos indivíduos e possibilitando tal manutenção (Marinho, et al., 2015).

A educação em saúde bucal não deve ser responsabilidade unicamente dos cirurgiões-dentistas, a equipe de saúde como um todo também pode estar envolvida no processo educativo, além do importante envolvimento dos pais, professores e agentes comunitários (Dutra, 2009).

Desta forma a escola assume um papel importante no desenvolvimento de atividades preventivas, pois reúne crianças de idades similares, somada a possibilidade de exercer educação em saúde de forma continuada por meio da aplicação de métodos educativos constantes. Tais métodos são capazes de atingir os indivíduos pertencentes ao grupo de polarização, que mesmo com os níveis da doença cárie em declínio no Brasil, ainda sofrem as decorrências da doença de forma mais acentuada, visto a influência de fatores socioeconômicos, demográficos e socioeducativos (Pinto, et al., 2018). Em síntese, a experiência de doença cárie sofre redução nos níveis de prevalência, porém atinge a população de forma desigual (Agnelli, 2016).

Verifica-se uma urgência no incentivo a políticas públicas com ênfase nos cuidados em saúde, em especial nos países em desenvolvimento, visto que essas medidas exibem a capacidade de promover saúde, prevenir doenças e amenizar as consequentes sequelas. Os espaços escolares são locais propícios para aplicação de tais medidas, incluindo o fomento a escovação dentária diária e supervisionada, aplicação regular de fluoretos, instruções acerca da dieta não cariogênica, entre outras medidas (Stein, 2017). Vale ressaltar a importância do enfrentamento da exclusão social e o fomento de medidas sociais e econômicas capazes de equilibrar a distribuição de renda, direcionadas principalmente à população de maior vulnerabilidade. A experiência da doença cárie se mostra desigual, mesmo com a prevalência da doença em declínio, onde cerca de $20 \%$ dos escolares sofrem carga de $60 \%$ da doença (Narvai, et al., 2006).

O aproveitamento do espaço escolar junto a profissionais de outras áreas da saúde tem relevância no processo educativo em saúde bucal, pois exibe a capacidade de proporcionar encontros prévios e frequentes quando comparados aos encontros com os cirurgiões-dentistas, visto que comumente são procurados após a instalação da doença. Nestes espaços pode-se oferecer informações básicas sobre aleitamento materno e suas influências no sistema estomatognático, higienização bucal e orientações inerentes a dieta não cariogênica, sobretudo ao aleitamento noturno como principal fator etiológico da cárie de acometimento precoce (Stein, 2017; Tinanoff, et al., 2019).

O presente estudo buscou avaliar o papel da escola na educação em saúde bucal de crianças em idade escolar, abrangendo toda a equipe pedagógica.

\section{Metodologia}

Trata-se de um estudo descritivo realizado no Espaço de Desenvolvimento Infantil Cidade de Lídice, localizada no bairro da Taquara no Rio de Janeiro. Fizeram parte do estudo a equipe educacional da escola, que inclui a direção da escola, professoras, inspetoras pertencentes ao cenário de prática acadêmica, supervisionadas pela preceptora local, da qual também participou do estudo. Participaram do estudo apenas os que aceitaram o convite de forma voluntária, por meio da assinatura do termo de consentimento livre e esclarecido (TCLE).

O estudo visou realizar uma pesquisa exploratória na escola, a fim de obter dados por meio da aplicação de questionários no formato de entrevista estruturada. As perguntas foram formuladas pelos integrantes do projeto abordando os seguintes assuntos: o papel da escola no processo educativo, métodos educativos em saúde, medidas básicas no controle da doença cárie e 
orientações de saúde bucal. Os dados qualitativos obtidos dos assuntos propostos foram tabulados dando à pesquisa dados qualiquantitativos permitindo a análise do nível de conhecimento da amostra. Após o preenchimento dos dados, foram transportados para o aplicativo Google formulários, seguido da criação de uma planilha e gráficos fornecidos pelo próprio software. (Pereira, et al., 2018)

Esse estudo é o resultado da aplicação do projeto de Extensão Oferta de cuidados em saúde bucal com ênfase em educação em saúde e higiene oral, aprovado pelo comitê de ética e pesquisa da Universidade do Grande Rio Professor José de Souza Herdy - UNIGRANRIO (CAAE 73211717.9.0000.5283).

\section{Resultados}

Aceitaram participar do presente estudo 11 voluntários da equipe educacional: que incluem a direção da escola, professoras, inspetoras e preceptora local.

Os resultados mostraram a grande sensibilidade da equipe educacional ao que diz respeito sobre o papel da escola no processo educativo em saúde bucal. Quando perguntados se concordam que a escola pode desempenhar um papel importante na oferta de informações sobre cuidados bucais para as crianças que nela estudam e se a contínua oferta de informação seria capaz evitar problemas bucais nas crianças, $100 \%$ responderam afirmativamente para as duas perguntas.

Cerca de 90\% dos entrevistados acreditam que há uma necessidade de a escola ser parceira dos pais na instrução higiene bucal de seus filhos e 9,1\% acreditam ser responsabilidade exclusiva dos pais ou responsáveis.

Quando indagados sobre quais seriam os melhores métodos de aprendizagem a serem utilizados para educação em saúde, puderam fazer mais de uma opção. Para processos educativos em adultos (Figura 1a), 90,9\% dos entrevistados elegeram como principal método as palestras, seguido do uso de panfletos por 36,4\% dos entrevistados.

Ao serem questionados sobre os métodos mais adequados para serem implementados em crianças (figura 1b), 90,9\% dos entrevistados selecionaram que a oferta de informações em saúde por meio de teatros representaria a forma mais adequada para aplicação do processo educativo, seguido, em igual proporção, das brincadeiras e historinhas $(72,7 \%$ dos entrevistados da equipe educacional). As metodologias que incluem o lúdico foram majoritariamente escolhidas, desta maneira seria possível atingir uma faixa etária mais abrangente e de forma agradável, deixando de lado a imagem da escovação dentária como uma obrigação punitiva. 
Figura 1 (a, b) — Gráficos referentes à pesquisa aos meios de informações sobre educação em saúde Figura 1a

De que forma vocé gostaria que essas informaçóes fossem passadas para o público em geral? 11 reapoutat

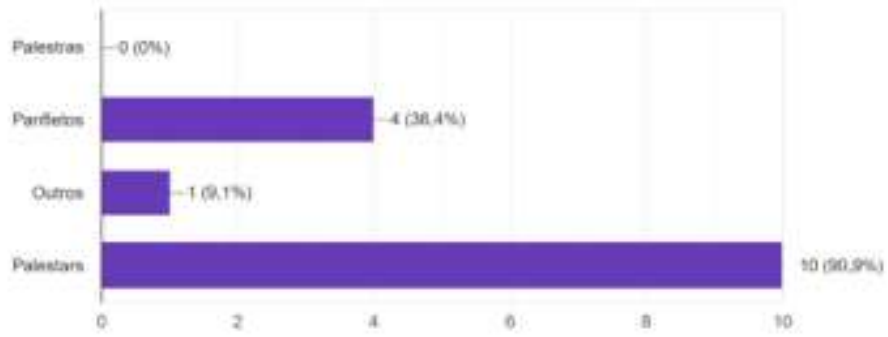

Figura tb

De que forma vocé gostaria que essas informaçరeses fossem passadas para crianças? 11 reapestas

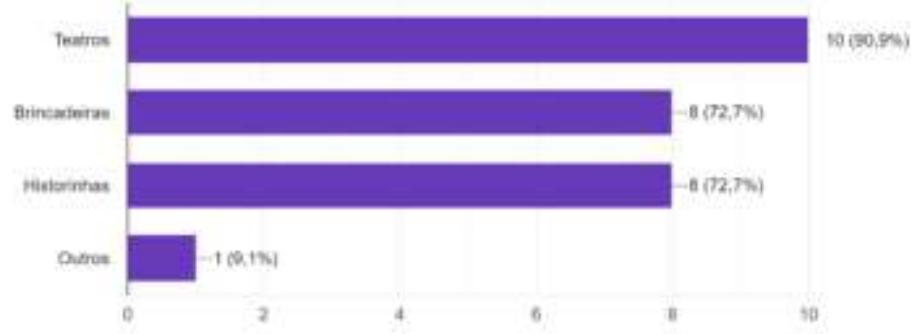

Fonte: Elaboração pelo google forms com base nos dados da pesquisa.

Os resultados obtidos foram expressivos ao constatar que 100\% dos voluntários já haviam sido consultados por um cirurgião-dentista ao menos uma vez. O tempo decorrido da visita ao dentista variou de 1 ano (54,5\%), 2 anos (27,3\%) e, 3 anos $(18,2 \%)$ (Figura 2a).

Quanto às medidas básicas de controle da doença cárie 100\% informaram incluir na rotina de saúde bucal, a escovação dos dentes. A porcentagem da frequência da escovação dentária foi de 54,5\% três vezes ao dia, seguido de 36,4\% duas vezes ao dia e $9,1 \%$ quatro vezes ao dia (Figura $2 b$ ). 
Figura 2 (a, b) — Gráficos referente à pesquisa sobre acesso aos cuidados bucais.

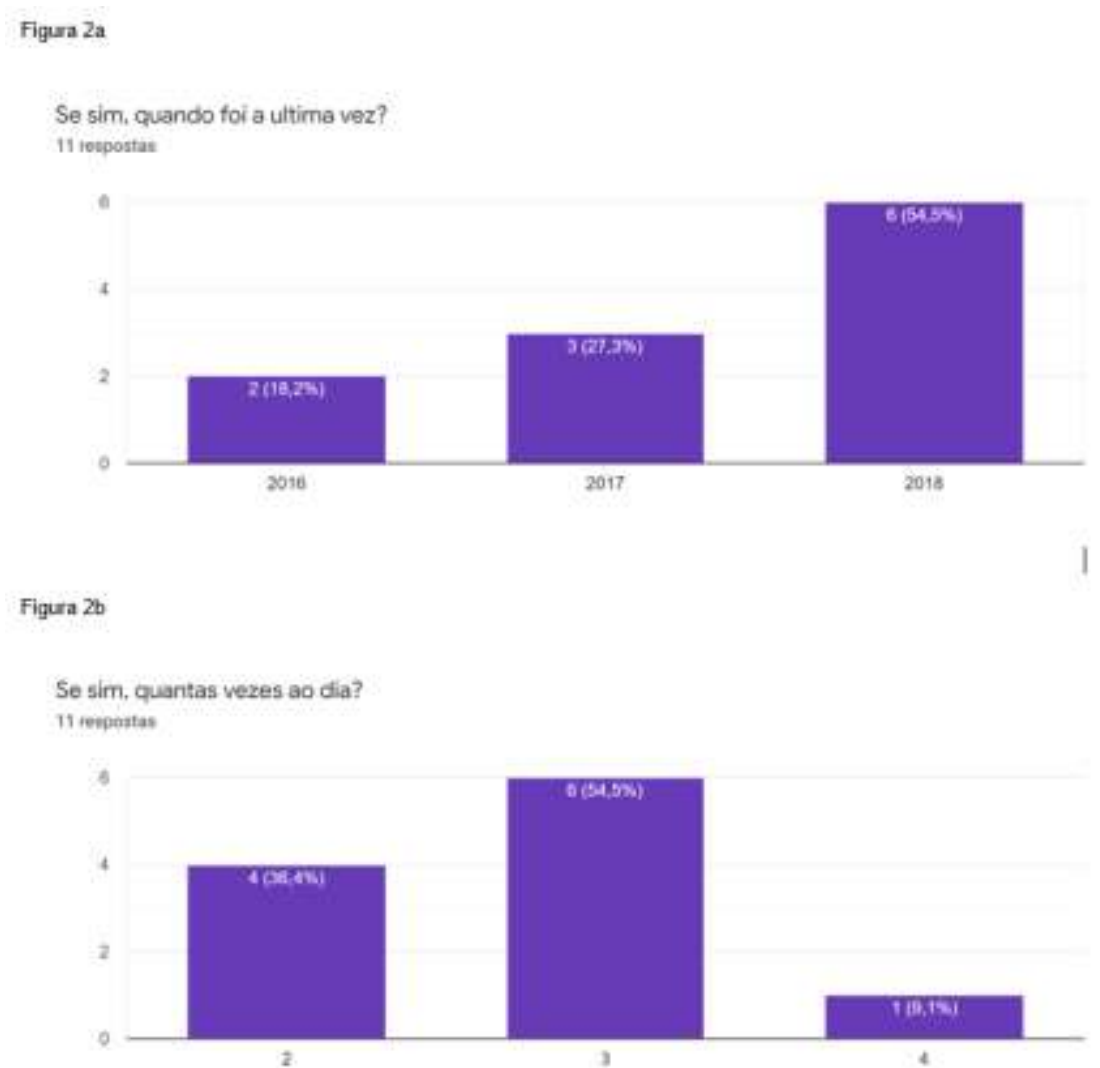

Fonte: Elaboração pelo google forms com base nos dados da pesquisa.

O uso do fio dental também faz e deve fazer parte desta rotina: 90,9\% expressam fazer o uso deste método (figura 3a). Dos entrevistados que afirmaram o questionamento anterior, $90 \%$ utilizam o fio dental pelo menos uma vez ao dia (figura $3 \mathrm{~b}$ ).

Sobre as orientações de higiene bucal, cerca de 90,9\% dos entrevistados já receberam orientações, através de dentista, familiares e outros profissionais da saúde (Figura 3c/d). 
Figura 3 (a, b, c, d) — Gráficos referentes à pesquisa de acesso aos cuidados bucais.

Figura 3a

Você usa fio dental?

11 respostas

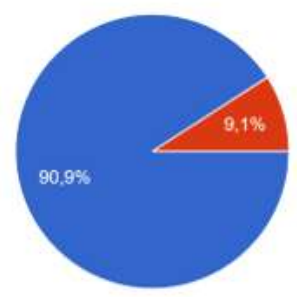

Figura 3c

Alguem ja Ihe explicou como escovar os dentes? 11 respostas

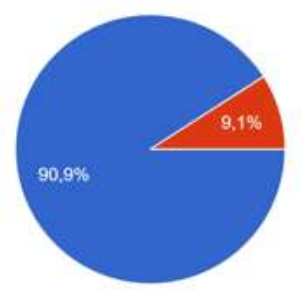

Figura 3b

$$
\begin{aligned}
& \text { Se sim, quantas vezes ao dia? } \\
& 10 \text { respostas }
\end{aligned}
$$

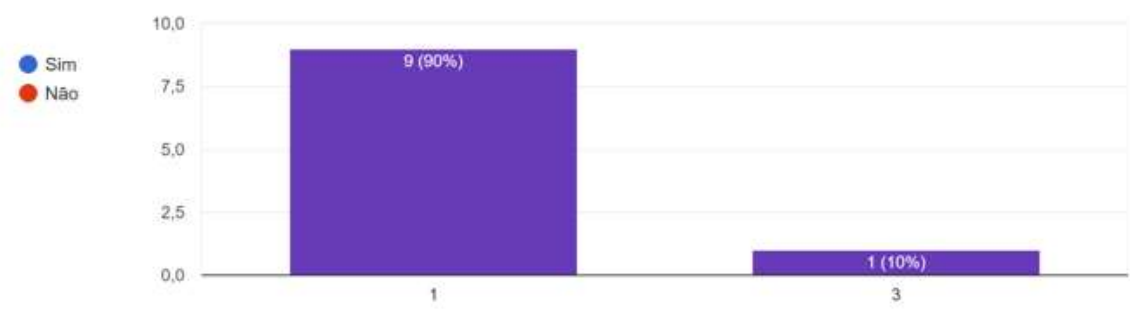

Figura 3d

$$
\text { Quem? }
$$

10 tespostas

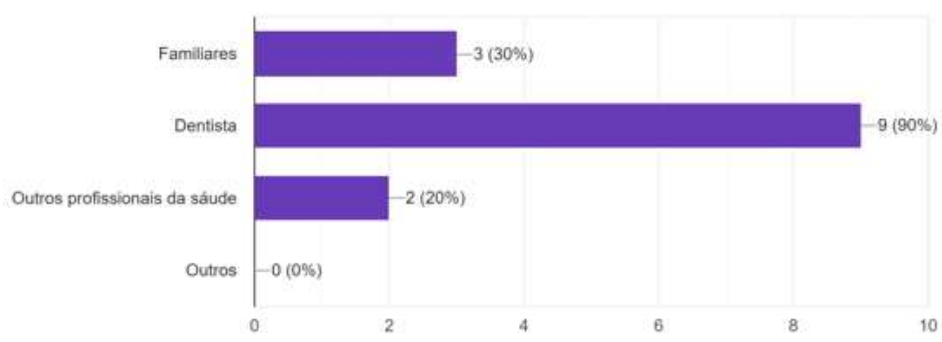

Fonte: Elaboração pelo google forms com base nos dados da pesquisa.

Em relação aos conhecimentos previamente adquiridos sobre 'O que é cárie dental?', cerca de $81,8 \%$ dos entrevistados responderam ser uma doença, 27,3\% selecionaram a opção "bichinhos no dente" e cerca de 9\% diziam ser um "buraco" no dente (Figura 4a). Após serem perguntados quanto ao que causaria cárie, os participantes puderam escolher mais de uma alternativa, onde a totalidade (100\%) disseram ser a ausência de escovação dentária, $72 \%$ responderam comer doces, 63,6\% beber refrigerantes, 45,5\% utilização de muitos antibióticos na infância e 18,2\% dentes enfraquecidos (Figura 4b).

Quanto ao controle/combate à cárie dental, de forma integral (100\%) responderam dentro das alternativas escovar os dentes (100\%), seguidos de $90,9 \%$ o uso do fio dental, $54,5 \%$ fazer bochecho e $36,4 \%$ escolher o que comer (Figura $4 \mathrm{c}$ ). 
Figura 4 (a, b, c) — Gráficos referentes à pesquisa de acesso aos cuidados bucais.

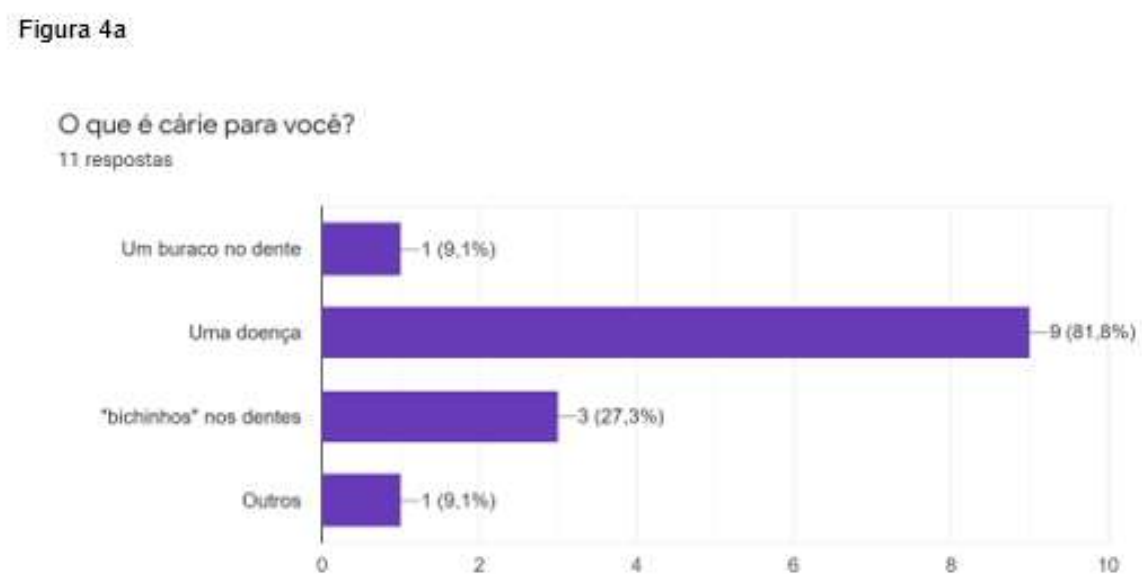

Figura $4 b$

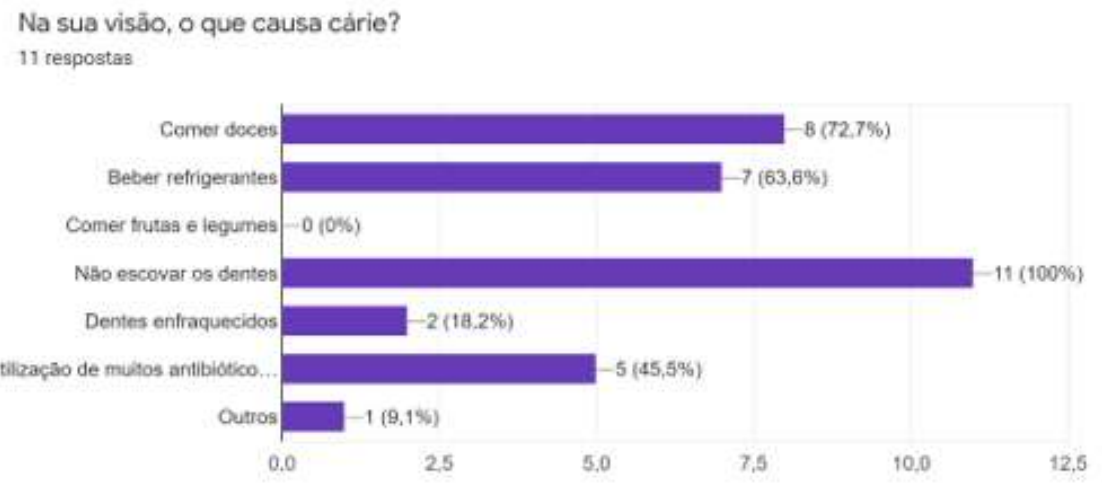

Figura $4 c$

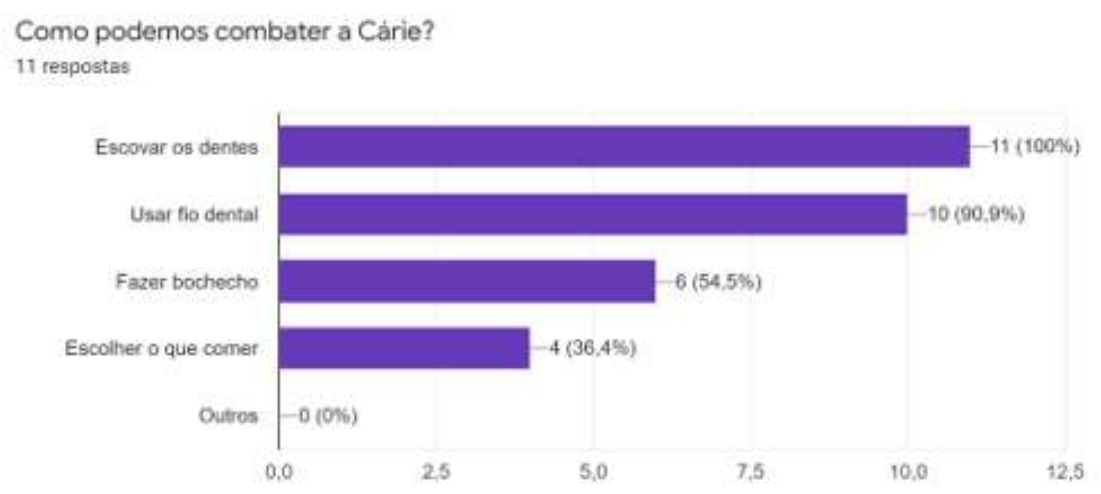

Fonte: Elaboração pelo google forms com base nos dados da pesquisa.

\section{Discussão}

Pauleto e colaboradores ressaltaram que as informações inerentes aos cuidados em saúde bucal devem atingir todas as camadas populacionais, de tal forma que os indivíduos consigam se tornar autônomos (Pauleto, et al., 2004). Em complemento, o autor ressaltou sobre a importância dos programas educativos, que vão de encontro às necessidades das populações mais vulneráveis que têm menor acesso aos serviços de saúde odontológicos, estes programas precisam ser valorizados e fomentados 
(Pauleto, et al., 2004; Duangthip, et al., 2017). Desta forma buscou-se desempenhar um papel educativo voltados às crianças de escolas públicas com o auxílio de professores e da direção escolar, atuando como "multiplicadores da saúde", identificando meios adequados e planejando abordagens diversas que permitissem a manutenção de hábitos saudáveis, com foco na prevenção das doenças cárie e periodontais.

É notório que a escola desempenha um papel importante na oferta de cuidados bucais para as crianças que nela estudam e que a inserção desses cuidados são capazes de prevenir ou reduzir os problemas bucais nas crianças. Cerca de $90 \%$ dos entrevistados acreditam que a escola deve atuar sendo parceira, em conjunto com os pais, na higiene bucal de seus filhos. No relatório político-pedagógico, Almeida e colaboradores puderam reconhecer o ambiente escolar como território fertil para desenvolver ações de promoção da saúde (Almeida, et al., 2020). Neste sentido, o estudo de Oliveira e colaboradores abordam sobre a necessidade de criar subsídios que incluam os educadores como facilitadores e multiplicadores do conhecimento, incluindo informações sobre higiene oral e cuidados mínimos para possibilitar a manutenção da saúde (Oliveira, et al., 2019), ainda nessa lógica, Frazão e colaboradores concluíram que as práticas de promoção de saúde bucal nas escolas públicas, independente do método adotado, exibiram a capacidade de beneficiar parte da população infantil mais vulnerável (Frazão, et al., 1996).

Segundo o Ministério da Saúde do Brasil a educação em saúde está compreendida nas ações de promoção e proteção à saúde, que tem como principal objetivo a obtenção de conhecimentos no tocante do processo saúde-doença, incluindo seus fatores de risco e proteção à saúde bucal, dando prioridade a construção da autonomia do indivíduo, atento às individualidades culturais. Vale ressaltar que as atividades do CD não se limitam apenas às ferramentas tecnicistas, é de competência do dentista realizar o planejamento, manutenção, avaliação e organização dessas atividades (Costa, et al., 2004). A intersetorialidade diz respeito ao envolvimento de múltiplos setores do indivíduo no cuidado em saúde, isto inclui escolas, local de trabalho, governo, mídia, entre outros, quanto mais setores engajados maior a efetividade das ações de promoção à saúde (Costa, et al., 2004), de tal forma que fica evidente a real importância do envolvimento da escola no processo saúde-doença.

Pode-se observar que o primeiro contato com orientações básicas de saúde advém de profissionais não ligados essencialmente a odontologia, como por exemplo, pediatras e enfermeiros, e estes profissionais exibem grande potencial na oferta das primeiras orientações quanto a importância da higienização bucal após o aleitamento materno noturno, práticas de higiene oral e orientação sobre a dieta não-cariogênica (Tinanoff, et al., 2019), o que reforça a importância da intersetorialidade, interdisciplinaridade e multiprofissionalismo.

Para Marinho e Monnerat algumas técnicas didáticas podem ser bem empregadas nas ações educativas em saúde, tais como palestras com emprego de fantoches, gincanas, montagem de cartazes, dramatização e apresentação de vídeos (Marinho, et al., 2015). Meneses e colaboradores realizaram uma revisão sistemática e observaram tamanha carência nos estudos sobre o emprego da ludicidade em saúde bucal e certa heterogeneidade (Meneses, et al., 2021), corroborando com Stein e colaboradores que apesar de considerarem evidentemente importante a educação em saúde, alerta o caráter urgente para o aumento de esforços em publicações que venham a comprovar a relevância do processo educativo em saúde (Stein, et al., 2017), de tal forma que seja possível evidenciar, com grande aporte de estudos científicos, os benefícios da prática educativa no combate à doença cárie, principalmente no grupo de polarização. O ministério da saúde brasileiro esclarece que esses conteúdos devem ser pedagogicamente projetados e reforça o bom rendimento de debates, oficinas de saúde, conversas em grupos, entre outros (Costa, et al., 2004).

Os resultados relativos à rotina de higiene oral foram satisfatórios no presente estudo, onde a totalidade dos entrevistados afirmaram realizar a escovação dentária, corroborando com os resultados obtidos no estudo de Stein e colaboradores, no qual observaram que $100 \%$ da amostra assumiram realizar a escovação dentária (Stein, et al., 2017). No que diz respeito a frequência dessa prática, Oliveira e colaboradores observaram que 45,1\% escovavam seus dentes quatro vezes ao dia (Oliveira, et al., 2019), 
este dado traduz que a prática e frequência da escovação é presente no dia a dia do indivíduo, mas como abordado anteriormente, a doença cárie é multifatorial e não somente a escovação dentária, mas outras atitudes devem estar presentes na rotina do paciente.

Tinanoff e colaboradores realizaram uma revisão sistemática e a partir dos dados obtidos foi possível observar diferenças significativas na alteração do índice de placa, favorecendo a hipótese de que a educação em saúde resulta de forma benéfica. Estes autores também realizaram a análise de outros estudos de teor semelhante, cujos resultados apresentados não alcançaram a mesma eficácia a curto prazo, o que pode ser justificado pelo número reduzido de participantes no grupo estudado (Tinanoff, et al., 2019). Ainda sobre a eficácia dos programas de saúde bucal em escolas, Aquilantes e colaboradores obtiveram resultados satisfatórios em pesquisa, onde houve um aumento de $16 \%$ nos conhecimentos sobre saúde bucal de crianças e uma diminuição de 0,63 no índice médio de placa e assim puderam concluir que a educação em saúde se torna importante para promover o aumento dos conhecimentos sobre saúde e reduzir o índice de placa (Aquilantes, et al., 2003), outros estudos observaram o impacto da educação em saúde no índice de higiene oral simplificado (IHOS), de Ferreira e colaboradores puderam concluir que após quatro semanas de atividades educativas houve redução do IHOS de 2,09 (inicial) para 1,79 (final) (de Freitas Oliveira, 2019), Ribeiro e colaboradores apontaram, a partir de análises, que houve efetividade da intervenção educativo-motivacional baseada na redução significativa da presença de biofilme $(p=0,028)$ (Barros, et al., 2020).

Ciente de que a doença cárie é passível de prevenção, torna-se premente estimular ações globais que atinjam não apenas a população geral, mas também que também englobem os indivíduos vulneráveis e suas particularidades, uma vez que não conseguem se enquadrar aos equilibrados níveis de controle da cárie dental. Locais como escolas são úteis tendo em vista não apenas a idade das crianças, mas também a frequência em que utilizam o serviço. A introdução ou a agregação de novos hábitos se torna tangível em crianças de idade escolar, somada a periodicidade em que ações educativas em saúde podem se dar no ambiente. Os efeitos ao conceder real importância aos ambientes escolares como propulsores de educação em saúde são comprovadamente satisfatórios, cujo prognóstico é extremamente favorável a médio e longo prazo, incluindo melhoras nos índices de controle da doença.

Vale ressaltar que estratégias locais, individualmente, não são suficientes para reduzir a concentração desigual da experiência da doença cárie no Brasil, esse resultado está atrelado também a melhor distribuição de renda e a justiça social, tendo em vista que quanto maior a desigualdade social maior a carga da doença cárie no grupo de polarização (Narvai, et al., 2006). Costa e colaboradores consideram que o Estado deve reconhecer e atuar nas desigualdades, priorizando aqueles com qualidade de vida insatisfatória e assim reduzir as disparidades em saúde (Costa e colaboradores, 2013), no estudo de Nery e colaboradores observou-se contrastes nos percentuais de promoção de saúde bucal em escolas nas cinco regiões do Brasil, a escola como promotor de saúde teve maior percentual nas regiões Sul e Sudeste, que apresentam melhores índices socioeconômicos, em comparação às regiões Centro-Oeste, Nordeste e Norte que possuem menores índices socioeconômicos (Nery, et al., 2019), este dado evidencia a relevância de tratar a saúde e os fatores sociais influenciadores. Estudos internacionais apontam a mesma problemática, no estudo de Kaewkamnerdpong e colaboradores foi observado que a alfabetização em saúde pôde reduzir $91 \%$ do risco de cárie em escolares que detém boa condição socioeconômica, porém o mesmo percentual não é observado em crianças em condições contrárias, indicando que a melhora nos índices de saúde bucal baseados na promoção de saúde é, também, determinado pelo fator social, em especial a equidade (Kaewkamnerdpong, 2018).

\section{Conclusão}

Como resultado deste trabalho, foi possível concluir que a escola desempenha um papel importante na educação em saúde bucal, e atua como uma importante ferramenta de políticas públicas para o controle e combate às doenças cárie e periodontais, tornando-se indispensável, em especial no grupo de polarização. Todavia, são necessários mais estudos de acompanhamento a longo prazo, possibilitando apresentar resultados significativos quanto ao benefício da educação em saúde, 
intervindo diretamente nos índices da doença, e, desta forma comprovar estatisticamente a redução dos impactos nos grupos de maior acometimento mediante à estas ações.

Espera-se que a partir deste estudo piloto, outros estudos possam ser desenvolvidos para que se possa observar os efeitos das ações de educação em saúde nas escolas.

\section{Referências}

Agnelli, P. B. (2016). Variação do índice CPOD do Brasil no período de 1980 a 2010. Revista Brasileira de Odontologia,72(1/2), 10

Aquilante, A. G., Almeida, B. S., Martins de Castro, R. F., Xavier, C. R. G., Sales Peres, S. H. C., \& Bastos, J. R. M. (2003). A importância da educação em saúde bucal para pré-escolares. Revista de Odontologia da UNESP, 32(1), 39-45.

Barros, L. R. C. Gregorio, D., Yokoyama, M. F., Hapner, A. V. P., Seixas, G. F., Neto, P. C., \& Maciel, S. M. (2020). Impacto de Ações Educacionais Sobre o Índice de Higiene Bucal de Escolares de um Município do Sul do Brasil. Ensaios e Ciência C Biológicas Agrárias e da Saúde, 24(3), 211-218.

Costa, H., Solla, J., Suassuna, A., \& Pucca Jr, G. A. (2004). Diretrizes da política nacional de saúde bucal. Brasília, Ministério da Saúde.

Costa, S. de M., Abreu, M. H. N. G. de, Vasconcelos, M., Lima, R. C. G. S., Verdi, M., \& Ferreira, E. F. (2013). Desigualdades na distribuição da cárie dentária no Brasil: uma abordagem bioética. Ciência \& Saúde Coletiva, 18(2), 461-470.

Almeida, L. E. de, Oliveira, A. B. A. de, Andrade, A. C. S., Peters, A. K., Floriano, A. L. R., Pestana, A. P. M., \& Bomtempo, C. C. (2020). Percurso políticopedagógico para o planejamento estratégico de uma ação de educação em saúde bucal vivenciada em um ambiente escolar. Cataventos-Revista de Extensão da Universidade de Cruz Alta, 12(1), 22-36.

Oliveira, M. F. de, Zanchett, S., \& Oliveira, A. W. C. (2019). Avaliação do índice de placa visível antes e depois de sessões de educação em saúde bucal com crianças. Revista de Atenção à Saúde, 17(60), 37-46.

Meneses, P. V. de S., Barbosa, É. P., Wanderley, F. A. C., \& Bandini, C. S. M. (2021). Atividades lúdicas para promoção de saúde bucal em escolares: revisão de literatura. Revista Eletrônica Acervo Saúde, 13(2), e5726-e5726. Doi: https://doi.org/10.25248/reas.e5726.2021

Duangthip, D., Chen, K. J., Gao, S. S., Lo, E. C. M., \& Chu, C. H. (2017). Managing early childhood caries with atraumatic restorative treatment and topical silver and fluoride agents. International journal of environmental research and public health, 14(10), 1204.

Dutra, I. R. (2009). Acesso e utilização aos serviços Atenção Primária à Saúde pela população urbana no município de Jequitinhonha, Minas Gerais.

Frazão, P., \& Narvai, P. C. (1996). Promoção da saúde bucal em escolas. Disciplina de Odontologia Preventiva e Saúde Pública: Manual do Aluno, 1-28.

Kaewkamnerdpong, I., \& Krisdapong, S. (2018). The associations of school oral health-related environments with oral health behaviours and dental caries in children. Caries research, 52(1-2), 166-175.

Monnerat, A. F. (2015). TRA-Tratamento Restaurador Atraumático: Abordagem Clínica em Saúde Pública-Conceito, Técnica, Tratamento e Materiais. Rio de Janeiro. Elsevier Brasil. p. 49-58.

Narvai, P. C., Frazão, P., Roncalli, A. G., \& Antunes, J. L. F. (2006). Cárie dentária no Brasil: declínio, polarização, iniqüidade e exclusão social. Revista Panamericana de Salud Pública, 19(6), 385-393

Nery, N. G., Jordão, L. M. R., \& Freire, M. do C. M. (2019). Ambiente escolar e promoção de saúde bucal: a Pesquisa Nacional de Saúde do Escolar (PeNSE). Revista de Saúde Pública, 53, 93.

Oliveira, E. L., Riatto, S. G., Vieira, A. P. S. B., Carvalho, G., Fonseca, M., Guedes, V., Pereira, J., \& Rocha, K. (2019). A importância do nível de conhecimento dos professores de escola pública do ensino fundamental sobre saúde bucal-revisão de literatura. Revista Campo do Saber, 4(5).

Pauleto, A. R. C., Pereira, M. L. T., \& Cyrino, E. G. (2004). Saúde bucal: uma revisão crítica sobre programações educativas para escolares. Ciência \& Saúde Coletiva, 9(1), 121-130.

Pinto, L. L., Ferreira, M. S., Araujo, T. L. C. de, \& Amaral, R. C. do (2018). Avaliação do conhecimento sobre a doença cárie de escolares do município de Juazeiro do Norte-CE. J. Health Sci. Inst, 23-27.

Stein, C., Santos, N. M. L., Hilgert, J. B., \& Hugo, F. N. (2018). Effectiveness of oral health education on oral hygiene and dental caries in schoolchildren: Systematic review and meta-analysis. Community dentistry and oral epidemiology, 46(1), 30-37.

Tinanoff, N., Baez, R. J., Guillory, C. D., Donly, K. J., Feldens, C. A., McGrath, C., Phatumvanit, P., Pitts, N. B., Seow, W. K., Sharkov, N., Songpaisan, Y., \& Twetman, S. (2019). Early childhood caries epidemiology, aetiology, risk assessment, societal burden, management, education, and policy: Global perspective. International journal of paediatric dentistry, 29(3), 238-248.

Pereira, A. S., Shitsuka, D. M., Parreira, F. J., \& Shitsuka, R. (2018). Metodologia da pesquisa científica. UFSM. https://repositorio. ufsm. br/bitstream/handle/1/15824/Lic_Computacao_Metodologia-Pesquisa-Cientifica. pdf. 